\title{
Selected parameters of the corneal deformation in the Corvis tonometer
}

\author{
Robert Koprowski ${ }^{1 *}$, Anita Lyssek-Boron ${ }^{2}$, Anna Nowinska², Edward Wylegala ${ }^{2}$, Henryk Kasprzak $^{3}$ \\ and Zygmunt Wrobel ${ }^{1}$
}

\author{
* Correspondence: robert. \\ koprowski@us.edu.pl \\ ${ }^{1}$ Department of Biomedical \\ Computer Systems, University of \\ Silesia, Faculty of Computer Science \\ and Materials Science, Institute of \\ Computer Science, ul. Będzińska 39, \\ Sosnowiec 41-200, Poland \\ Full list of author information is \\ available at the end of the article
}

\begin{abstract}
Introduction: Contemporary ophthalmology knows many methods of measuring intraocular pressure, namely the methods of non-contact and impression applanation tonometry. In non-contact applanation tonometers, e.g. the Corvis, the corneal flattening is caused by an air puff. Image registration of the corneal deflection performed by a tonometer enables to determine other interesting biomechanical parameters of the eye, which are not available in the tonometer. The measurement of new selected parameters is presented in this paper.
\end{abstract}

Material and method: Images with an $M \times N \times$ I resolution of $200 \times 576 \times 140$ pixels were acquired from the Corvis device in the source recording format *..cst. A total of 13 '400 2D images of patients examined routinely in the Clinical Department of Ophthalmology, in District Railway Hospital in Katowice, Poland, were analysed in accordance with the Declaration of Helsinki. A new method has been proposed for the analysis of corneal deflection images in the Corvis tonometer with the use of the Canny edge detection method, mathematical morphology methods and context-free operations.

Results: The resulting image analysis tool allows determination of the response of the cornea and the entire eyeball to an air puff. The paper presents the method that enables the measurement of the amplitude of curvature changes in the frequency range from 150 to $500 \mathrm{~Hz}$ and automatic designation of the eyeball movement direction. The analysis of these data resulted in 3 new features of dynamics of the eye reaction to an air puff. Classification of these features enabled to propose 4 classes of deformation. The proposed algorithm allows to obtain reproducible results fully automatically at a time of $5 \mathrm{~s}$ per patient using the Core i5 CPU M460 @ 2.5GHz 4GB of RAM.

Conclusions: The paper presents the possibility of using a profiled algorithm of image analysis, proposed by the authors, to measure additional cornea deformation parameters. The new tool enables automatic measurement of the additional new parameters when using the Corvis tonometer. A detailed clinical examination based on this method will be presented in subsequent papers.

Keywords: Eye biomechanics, Corvis tonometer, Image processing, Measurement Automation, Segmentation

\section{Biomed Central}

(c) 2014 Koprowski et al.; licensee BioMed Central Ltd. This is an Open Access article distributed under the terms of the Creative Commons Attribution License (http://creativecommons.org/licenses/by/4.0), which permits unrestricted use, distribution, and reproduction in any medium, provided the original work is properly credited. The Creative Commons Public Domain Dedication waiver (http://creativecommons.org/publicdomain/zero/1.0/) applies to the data made available in this article, unless otherwise stated. 


\section{Introduction}

Contemporary ophthalmology knows many methods of measuring intraocular pressure. These include non-contact and impression applanation tonometry. They are based on the Imbert-Fick law. Knowing the physical strength necessary to flatten a sphere and the surface of the flattening, it is possible to determine the pressure inside the sphere. Non-contact applanation tonometers, e.g. the Corvis, use this rule. In this tonometer, the flattening of the cornea is caused by an air puff - Figure 1. The device measures the cornea thickness, deflection amplitude, applanation length, corneal deflection speed, intraocular pressure. In addition, the information about places of applanation, pachymetry and other data are provided - Figure 2. The locations of applanation (temporary cornea flattening during a convex-concave transition and vice versa) enable to determine two applanation times $t_{1}$ and $t_{2}$. In Ocular Response Analyzer (ORA) the air pressure values $P_{1}$ and $P_{2}$ are read in applanation times $t_{1}$ and $t_{2}$, and then the value $\left(P_{1}+P_{2}\right) / 2$ is calculated. This value after linear calibration is the value of the intraocular pressure IOP. The Corvis tonometer also records sequence of images of the corneal deflection (resulting from the air puff). The sequence of images enables to get much more information using the methods of image analysis and processing. Such analysis allows to obtain three-dimensional reconstruction of corneal deflection in one of time ranges. On this basis, it is possible to determine the locations of applanation, the corneal deformation rate or the entire eyeball response to the air puff.

Analysis of images from the Corvis tonometer in the original software is based on designation of the outer and inner edges of the cornea profile. The edge detection methods of Roberts, Sobel and Prewitt can be all applied here. The Canny filter or local thresholding dependent on local brightness changes can be also used with equally good results. Additionally, the visible cross-sections of the iris which are often the brightest visible area are problematic here. All these methods based on a typical standard approach, enable to detect the corneal deformation in only one selected place (usually the main axis of fixation). From a biomechanical point of view, other locations of the

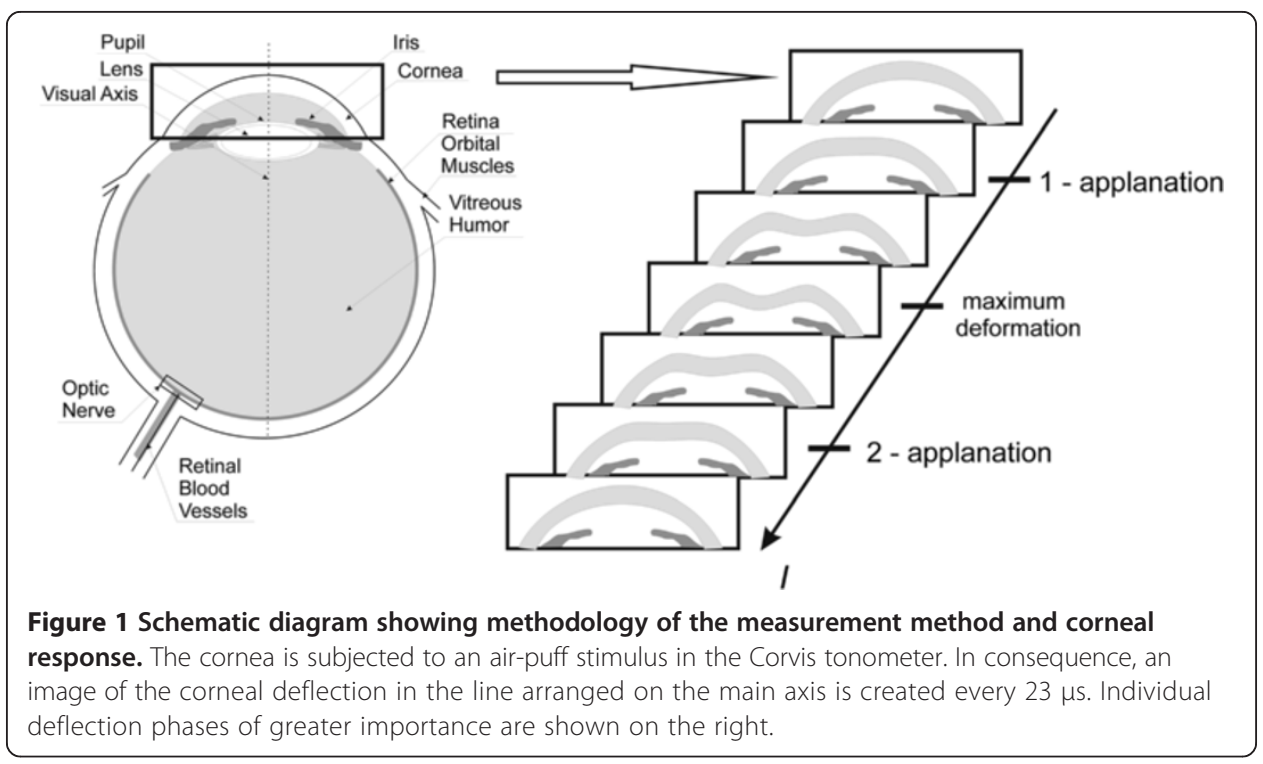




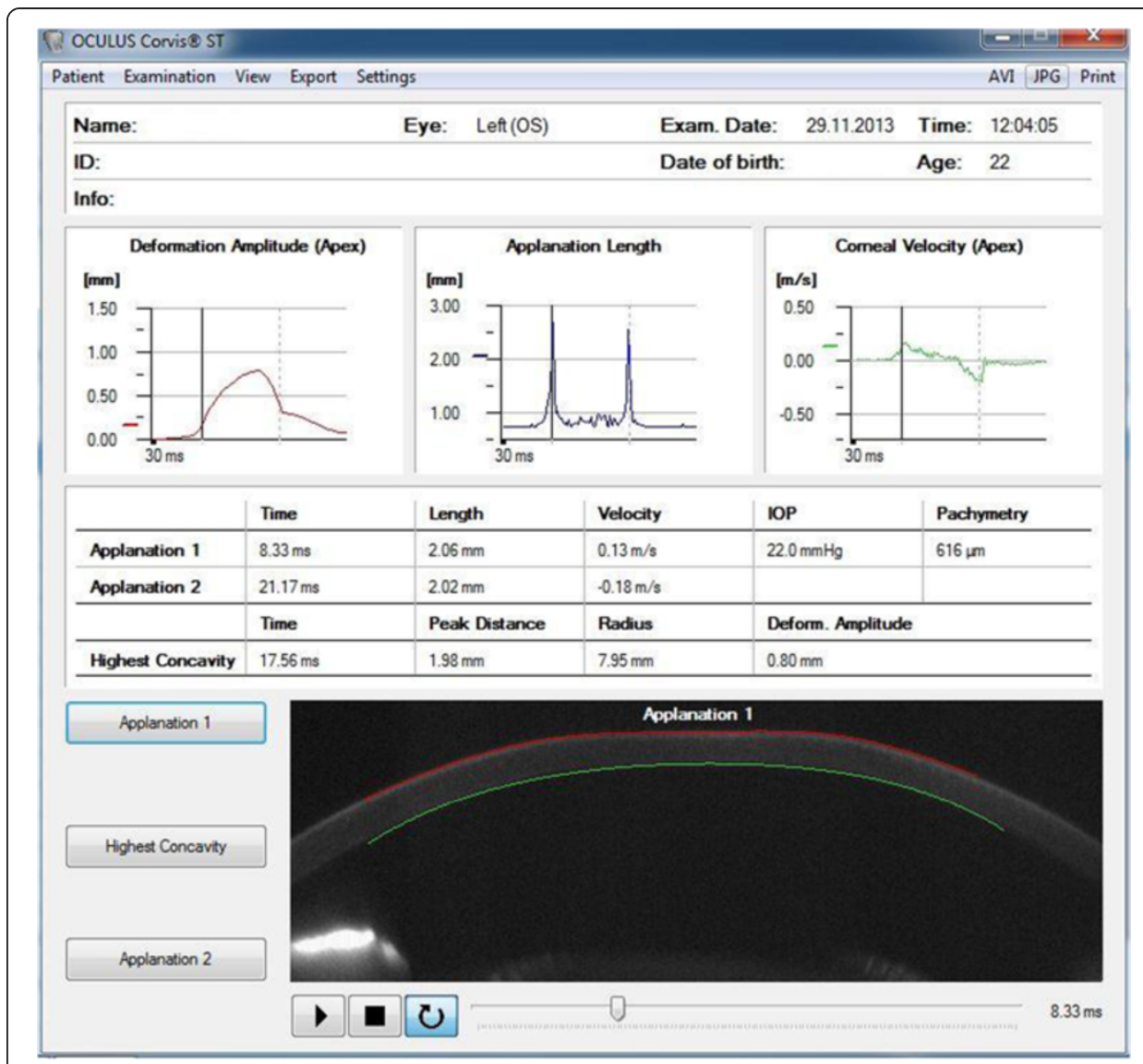

Figure 2 Examples of results obtained from the Corvis device. There are three graphs: deformation amplitude, applanation length, corneal velocity and two examples of applanation. For these two examples ( 1 and 2), time, length and velocity are given. On this basis and after linear calibration, the value of intraocular pressure IOP is determined.

corneal deformation or determination of the eyeball reaction to the air puff are also of interest. These parameters are not adequately described in the known publications in terms of both image analysis and processing as well as biomechanics.

The papers published so far are related to the analysis of corneal biomechanical parameters for the data derived mainly from the ORA device [1,2]. In [1], measurements of several populations indicate that corneal hysteresis, a biomechanical measure, varied over a dynamic range of 1.8 to $14.6 \mathrm{mmHg}$ and was only weakly correlated with corneal thickness $\left(r^{2}=0.12\right)$. This is related to the observation that some subjects with relatively thick corneas have less-than-average corneal hysteresis. For the cornea itself, the effect of the patient's age [3-6], glaucoma [7] or wound healing [8] on its biomechanical parameters is also considered. Predictive numerical simulation of corneal biomechanical parameters was considered in an equally interesting way in [9]. In this paper, twentyfour corneal buttons were tested under posterior inflation conditions while monitoring their behaviour using non-contact methods. The measurement methods of the ultrastructure of the corneal stroma were presented in [10]. There are also very interesting studies profiled to the cornea analysis of Brazilian patients [11] or children with the use of the ORA tonometer [12,13]. A group of authors in [14] presented a correlation 
between hysteresis generated in a tonometer and pachymetry. By contrast, in [15] the same hysteresis as in [16] was presented in correlation with the glaucoma damage. The paper [17] deals with these issues (hysteresis), but in the Reichert ORA device. There exists also a well-known group of papers concerning the analysis of biomechanical parameters of the cornea in keratoconic eyes [18] or LASIK [19]. Moreover, there are descriptions of other tests of the cornea which contribute to the understanding of the biomechanical properties such as the comparison of the corneal strip extensometry presented in [20].

Last year has appeared series of papers dealing with application of Corvis noncontact tonometer to examine both IOP value as well as biomechanical parameters of the cornea, related to the corneal deformation [21-24]. However, Corvis tonometer gives more possibilities of corneal measurements after application of additional algorithms and procedures of data processing.

Therefore, there is a need to propose a dedicated automatic algorithm enabling measurements performed at out of axis places of the corneal deformation or determination of the eyeball response to an air puff during pressure measurement. The proposed dedicated algorithm and the results obtained from its use are discussed in detail later in the paper.

\section{Material}

Images with an $M \times N \times I$ resolution of $200 \times 576 \times 140$ pixels were acquired from the Corvis device in the source recording format ".cst (they can be converted to a sequence of images *.bmp, ".jpg or a film *.avi). The patients ranging from 17 to 63 years of age were healthy (32 people including 16 women) and ill (16 people including 9 women). The group of ill patients suffered from either AMD or other diseases that cause abnormal pressure in the eye. A total of 96 eyes were examined and for each one a sequence of 140 images was obtained - which accounted for 13'400 2D images for analysis. The patients were examined during tests performed routinely in the Clinical Research Department of Ophthalmology, in District Railway Hospital in Katowice, Poland, in accordance with the Declaration of Helsinki. All the patient data have been anonymised.

\section{Method}

\section{Pre-processing}

Pre-processing of input images $L_{G R A Y}(m, n, i)$, where $m$-row $m \in(1, M), n$-column $n \in(1, N), i$ - another 2D image $i \in(1, I)$, is related to their automatic reading from the source file with the extension *.cst. In the file, "..cst is a sequence of $2 \mathrm{D}$ images preceded by a header which contains basic information about the patient. A sequence of images $L_{G R A Y}(m, n, i)$ thus obtained is then subjected to median filtering with a mask $h_{1}$ sized $M_{h 1} \times N_{h 1} \times I_{h 1}=3 \times 3 \times 3$ pixels. The mask size was chosen arbitrarily, taking into account the size of distortions and artefacts entering the optical path. In case of larger mask size $h_{1}$, median filtering was causing the deletion of the corneal contour, therefore the size $M_{h 1} \times N_{h 1} \times I_{h 1}=3 \times 3 \times 3$ pixels was optimal. The filtered image $L_{M}(m, n, i)$ undergoes further preliminary transformations. These include designation of the outer edge of the cornea. Two different independent approaches to determining the outer limit of the cornea were implemented. 
The first one involves analysis of brightness for each column of the image $L_{M}(m, n=$ const, $i=$ const $)$. Each column is subjected to binarization operation with a threshold set automatically according to the Otsu formula [25], i.e.:

$$
L_{B}(m, n, i)=\left\{\begin{array}{cc}
1 & \text { if } \\
0 & \text { other }
\end{array} L_{M}(m, n, i)>\left(v_{r} \cdot p_{r}(n, i)\right)\right.
$$

where: $p_{r}(n, i)$ - binarization threshold set automatically according to the Otsu formula for $m \in(1, M)$,

$v_{r}$ - automatic correction factor chosen arbitrarily at 0.5 .

According to formula (1) binarization threshold is determined on the basis of two values: $p_{r}(n, i)$ which is the binarization threshold according to the Otsu and $v_{r}$ which was chosen arbitrarily. Factor $v_{r}$ is the correction factor resulting from the image specificity $L_{M}(m, n, i)$. There is a necessity of the correction of the threshold value $p_{r}(n, i)$ by $50 \%(0.5)$ due to high contrast of the corneal edges occurring in some cases. The resulting binary image $L_{B}(m, n, i)$ is analysed to enable determination of the outer edge of the cornea. One of the simplest methods is the calculation of the XOR value between two images. $L_{B}(m, n, i)$ is one image and the other one is the result of its morphological erosion $L_{E}(m, n, i)=\min \left(L_{B}(m, n, i)\right)$ with a structural element $S E$ sized $M_{S E} \times N_{S E} \times I_{S E}=3 \times 3 \times 3$ pixels. It results in the matrix $L_{K}(m, n, i)$ :

$$
L_{K}(m, n, i)=\left(\min _{S E}\left(L_{B}(m, n, i)\right)\right) \underline{\vee}\left(L_{B}(m, n, i)\right)
$$

Since only the outer limit is determined, its contour $L_{P}{ }^{(1)}(n, i)$ can be defined as follows:

$$
\begin{aligned}
& L_{R}(m, n, i)=\left\{\begin{array}{cc}
m & \text { if } \\
0 & \text { other }
\end{array} \quad L_{K}(m, n, i)=1\right. \\
& L_{P}^{(1)}(n, i)=\left\{\begin{array}{cc}
\min _{m}\left(L_{R}(m, n, i)\right) & \text { if } \\
0 & \text { other }
\end{array}\right.
\end{aligned}
$$

The results are, to a large extent, influenced by the brightness of the iris cross-section which, in turn, affects the overvaluation of the threshold value $p_{r}$. As a result, the proper edge of the cornea is not properly recognized. For the analysed 13'400 2D images, the above method of edge detection works well in about $80 \%$ of cases. The value of $80 \%$ of cases was defined as the number of correctly detected corneal contours in all analyzed cases (13'400 2D images). The correctly detected corneal contour was described as not having any discontinuity points and the difference of the position of each contour point was below 10 pixels (maximum displacement of the contour line) between following series images.

The other approach involves using the well-known Canny edge detection method. The obtained result $L_{C}(m, n, i)$ of edge detection, for the arbitrarily selected parameters of the threshold (0.1) and standard deviation of the Gaussian filter (0.99), requires further correction. This adjustment only involves morphological closing, i.e.:

$$
L_{W}(m, n, i)=\min _{S E}\left(\max _{S E}\left(L_{C}(m, n, i)\right)\right)
$$

Next, the binary image $L_{W}(m, n, i)$ is subjected to determination of the upper edge analogously to (3), (4). The resulting contour of the outer edge of the cornea $L_{P}{ }^{(2)}(n, i)$ is correct 
in about $90 \%$ of cases for the analysed 13'400 2D images. The definition of the correctly detected corneal contours were analogous to the previously described method.

Incorrectly detected cornea outer edges coincide for both methods, $\left(L_{P}{ }^{(1)}(n, i)\right)$ and $\left.L_{P}{ }^{(2)}(n, i)\right)$, and for this reason they cannot be mutually complementary. Due to better edge detection in the case of the second method (Canny), it was used further $\left(L_{P}(n, i)\right.$ for simplification). The results are shown in Figure 3.

\section{Processing}

The outer edge of the cornea $L_{P}(n, i)$, determined in image pre-processing, forms the basis for further analysis. Automatic designation of two important parameters, namely the correction of the cornea deformation curve and the rapidly changing cornea deformation in dynamic states when applying force, is described below.

\section{Cornea deformation correction}

Under the influence of a pressure impulse from the Corvis tonometer, the cornea is deformed and the entire eyeball moves in the eye socket. For proper evaluation and interpretation of the results, displacement of the entire eyeball should be separated from the deflection curve of the cornea. For this purpose, in the first step, the shape of the cornea visible in time $t=0$ (for $i=1$ ) is removed, i.e.:

$$
L_{T}(n, i)=L_{P}(n, i)-L_{P}(n, 1)
$$

The resulting image $L_{T}(n, i)$ and the graph $L_{T}(N / 2, i)$ are shown in Figure 4. The graph $L_{T}(N / 2, i)$ consists of two parts of the response: corneal deflection $L_{T R}(N / 2, i)$ and the eyeball reaction $L_{T O}(N / 2, i)$ to an air-puff stimulus, in general:

$$
L_{T}(n, i)=L_{T O}(n, i)+L_{T R}(n, i)
$$

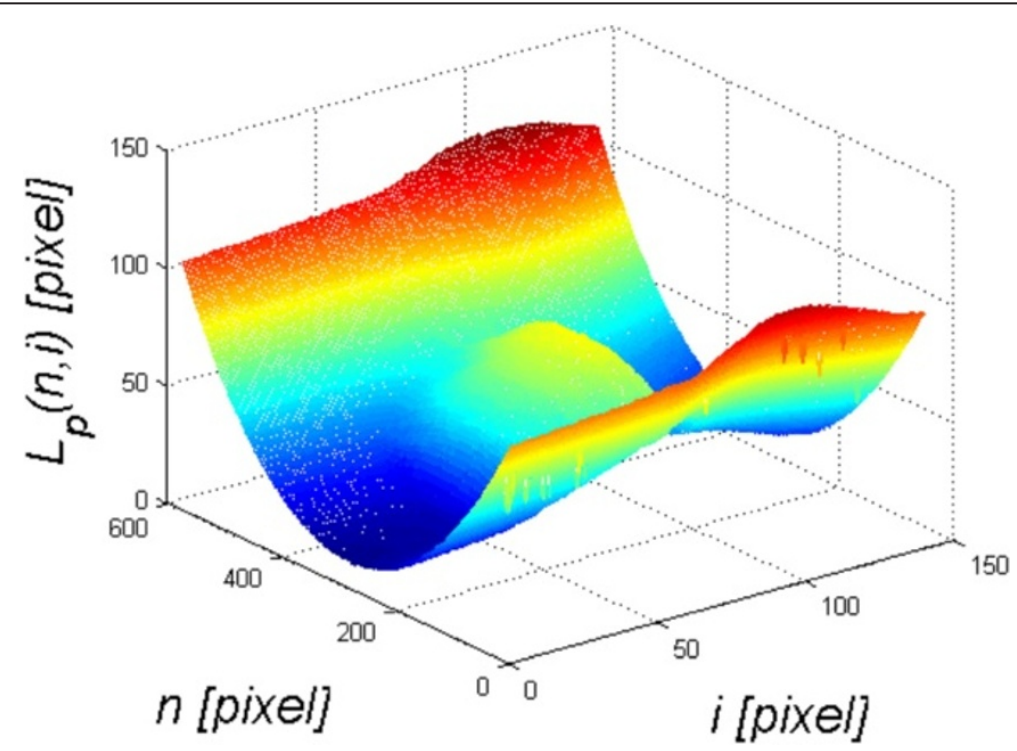

Figure 3 Example of a cornea outer edge detection result. The image $L_{p}(n, i)$ results from the Canny edge analysis and additional processing proposed by the authors. Local problems with edge detection can be removed here by median filtration with a mask sized $3 \times 3$ pixels. This image is further processed. 

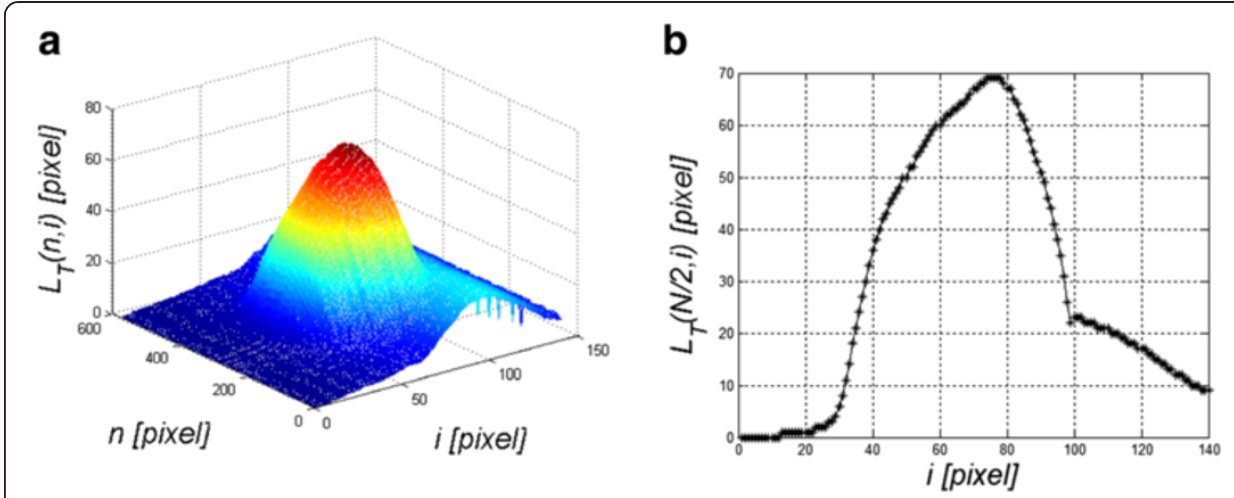

Figure 4 Sample graphs $L_{T}(n, i)$ and $L_{T}(N / 2, i)$. The results obtained concern a relative cornea deformation resulting from an air-puff stimulus. The cross-section along the main axis N/2 is shown on the left a). This graph consists of two overlapping waveforms. One refers to the cornea deformation and the other one to the entire eyeball response to an air puff. The object of the analysis is, inter alia, separation of these two waveforms - b).

The separation of these two waveforms $\left(L_{T R}(N / 2, i)\right.$ and $\left.L_{T O}(N / 2, i)\right)$ from $L_{T}(N / 2, i)$ is possible owing to the analysis of the visible contour of the cornea on the left and right borders of the image, i.e.:

$$
L_{T O}(n, i)=\frac{L_{T}(N, i)-L_{T}(1, i)}{N-1} \cdot(n-1)+L_{T}(1, i)
$$

The waveform $L_{T O}(n, i)$ at the point $N / 2$ defined with the formula (8) can be subtracted from $L_{T}(N / 2, i)$ (formula (7)). The correctness of the algorithm was pre-verified by determining the correlation between the maximum amplitude of deviation calculated by the Corvis software and the same quantity determined from the equation (7) - $\max \left(L_{T R}\right)$. The results obtained are shown in Figure 5 - the error calculated as the mean standard deviation is less than $1 \%$. Next, image analysis results are shown in Figure 6. These results (Figure 6) are extremely interesting from the point of view of biomechanics of the eye. The movement of the eyeball in response to a pressure impulse can be observed. As a result, it is possible to correct the waveform of the cornea deformation itself. Also the symmetry in the waveforms $L_{T R}(N / 2, i)$ and $L_{T O}(N / 2, i)$ is quite clearly visible - Figure 7 . Separation of the eyeball displacement $L_{T O}(n, i)$ from the waveform of the cornea deformation $L_{T}(n, i)$ allows to measure another new parameter - rapidly changing cornea deformations visible during force application.

\section{Rapidly changing cornea deformations during force application}

Rapidly changing cornea deformations (with a frequency above $150 \mathrm{~Hz}$ ) are directly visible in Figure 4. These deformations are not measured by the Corvis tonometer and they contain significant information about the biomechanical parameters of the eye (in particular the cornea). Their measurement is possible owing to the profiled image analysis algorithm.

The image $L_{T R}(n, i)$, resulting from the previous processing stage, undergoes an operation which involves the removal of a slowly changing deformation. For this purpose, the image resulting from morphological opening is subtracted from the image $L_{T R}(n, i)$, i.e.:

$$
L_{Q}(n, i)=L_{T R}(n, i)-\max _{S E 2}\left(\min _{S E 2}\left(L_{T R}(n, i)\right)\right)
$$




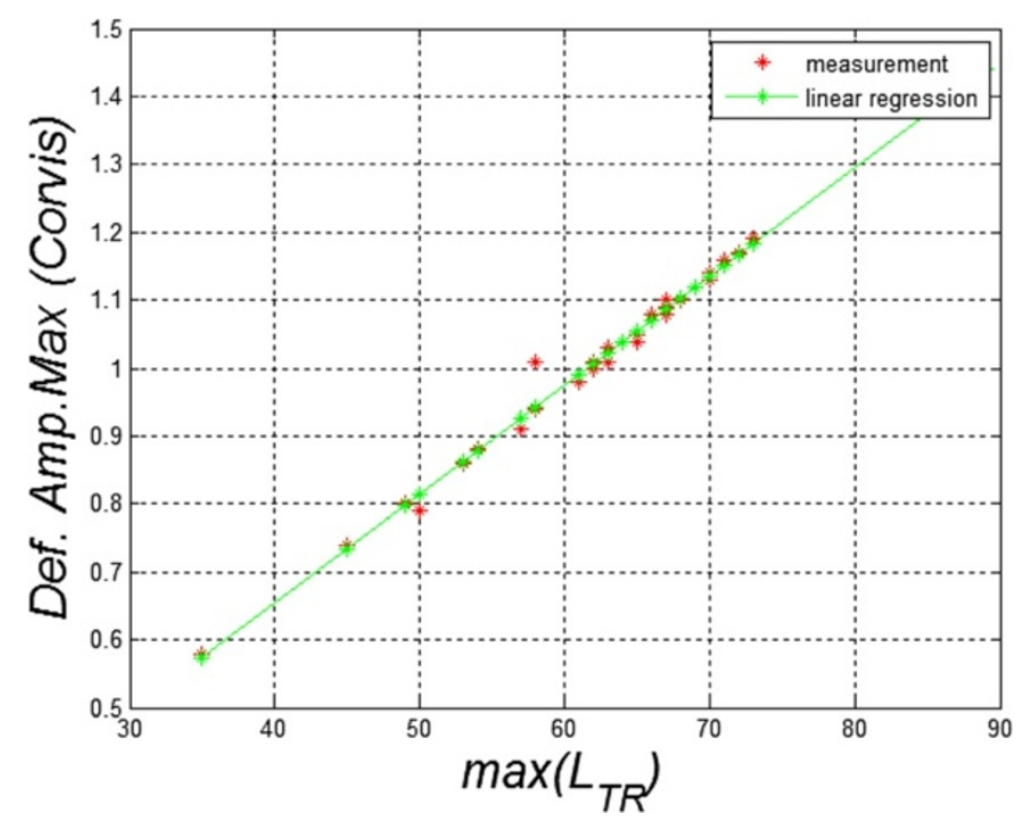

Figure 5 Correlation between the maximum amplitude of the cornea deformation calculated with the Corvis software and the author's algorithm. These results confirm the correctness of the adopted algorithm of analysis and processing of image sequences and their reading from the format *..cst. On this basis, other parameters described in the paper were further determined and calculated.

for a structural element $S E 2$ sized $N_{S E 2} \times I_{S E 2}=33 \times 33$ pixels. The size of the structural element $S E 2$ is matched to the low-frequency range, lasting more than a few dozen milliseconds. The resulting image $L_{Q}(n, i)$ is shown in Figure $8 \mathrm{a}$, whereas Figure $8 \mathrm{~b}$ presents the graphs $L_{Q L}(i)$ and $L_{Q R}(i)$. The results of FFT (Fast Fourier Transform), namely $F_{\mathrm{QR}}$ and $F_{\mathrm{Q} L}$, of the graphs $L_{\mathrm{Q} L}(i)$ and $L_{\mathrm{QR}}(i)$ are shown in Figure $8 \mathrm{c}$, whereas Figure $8 \mathrm{~d}$ presents individual components $L_{\mathrm{Q} L}{ }^{(I)}(i)$ and $L_{\mathrm{Q} L}{ }^{(I I)}(i)$ as well as their sum $L_{\mathrm{Q} L}{ }^{(I+I I)}(i)$ of the waveform $L_{Q L}(i)$. The individual components $L_{Q L}(i)$ and $L_{Q L}{ }^{(I I)}(i)$ were obtained from harmonics marked in Figure $8 \mathrm{c}$ with areas I and II. These are respectively: I- constant and slowly changing component (below $150 \mathrm{~Hz}$ ) and II- component responsible for periodic
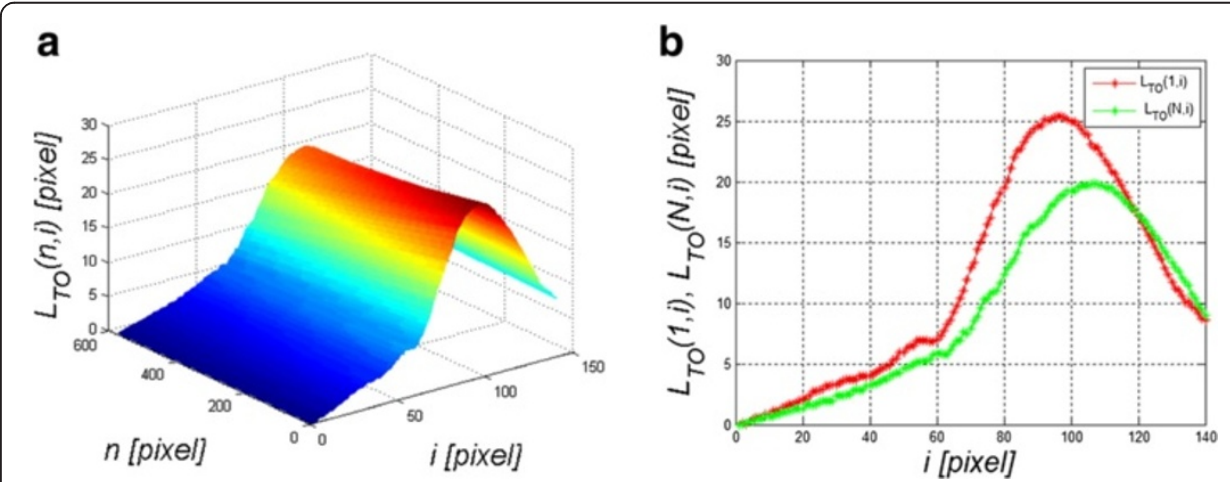

Figure 6 Examples of analysis results - the image $L_{T O}(n, i)$ is the eyeball displacement. Additionally, the graph on the right a) shows the waveforms $L_{T O}(1, i)$ and $L_{T O}(N, i)$. Using the methods of image analysis and processing described by the authors, the proper corneal deflection caused by an air puff can be separated from the eyeball displacement - b). 


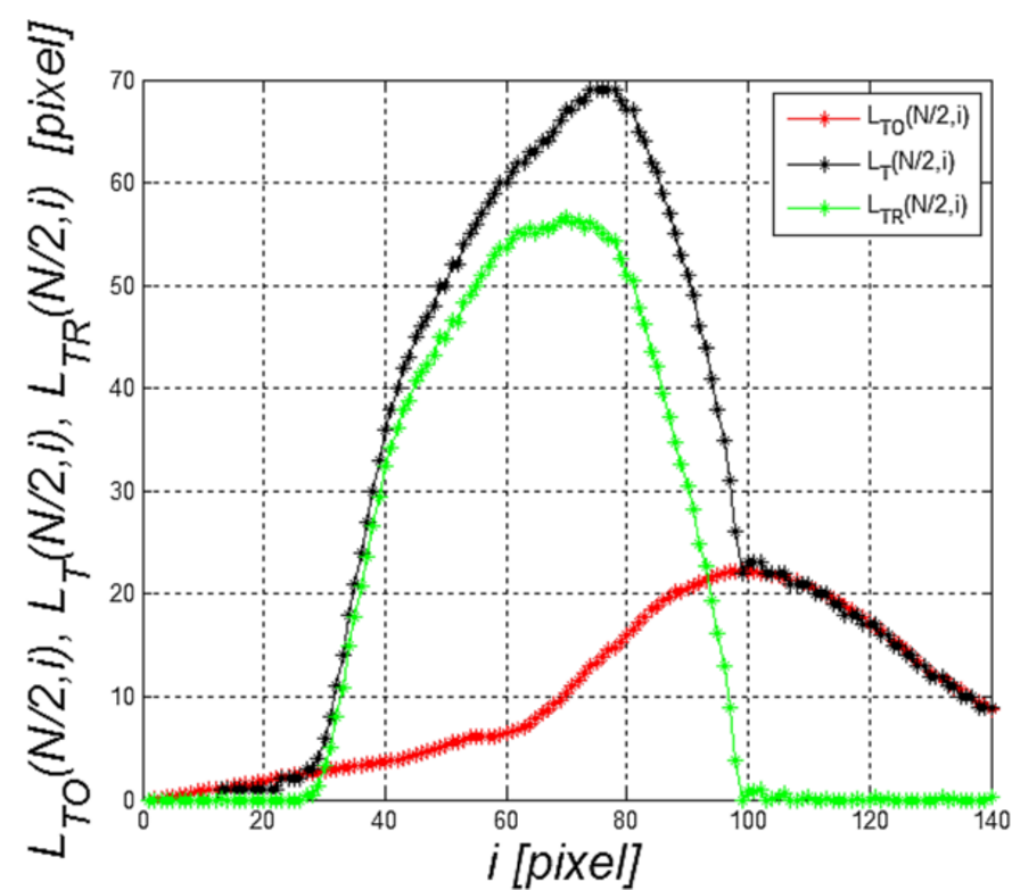

Figure 7 Waveform $L_{T}(n, i), L_{T O}(n, i)$ and $L_{T R}(n, i)$. Separation of the waveforms $\left(L_{T O}(n, i)\right.$ and $\left.L_{T R}(n, i)\right)$ enabled separation of the entire eyeball reaction from the cornea response to an air-puff stimulus. Therefore, further analysis and measurement of properties specific for each of them is possible.
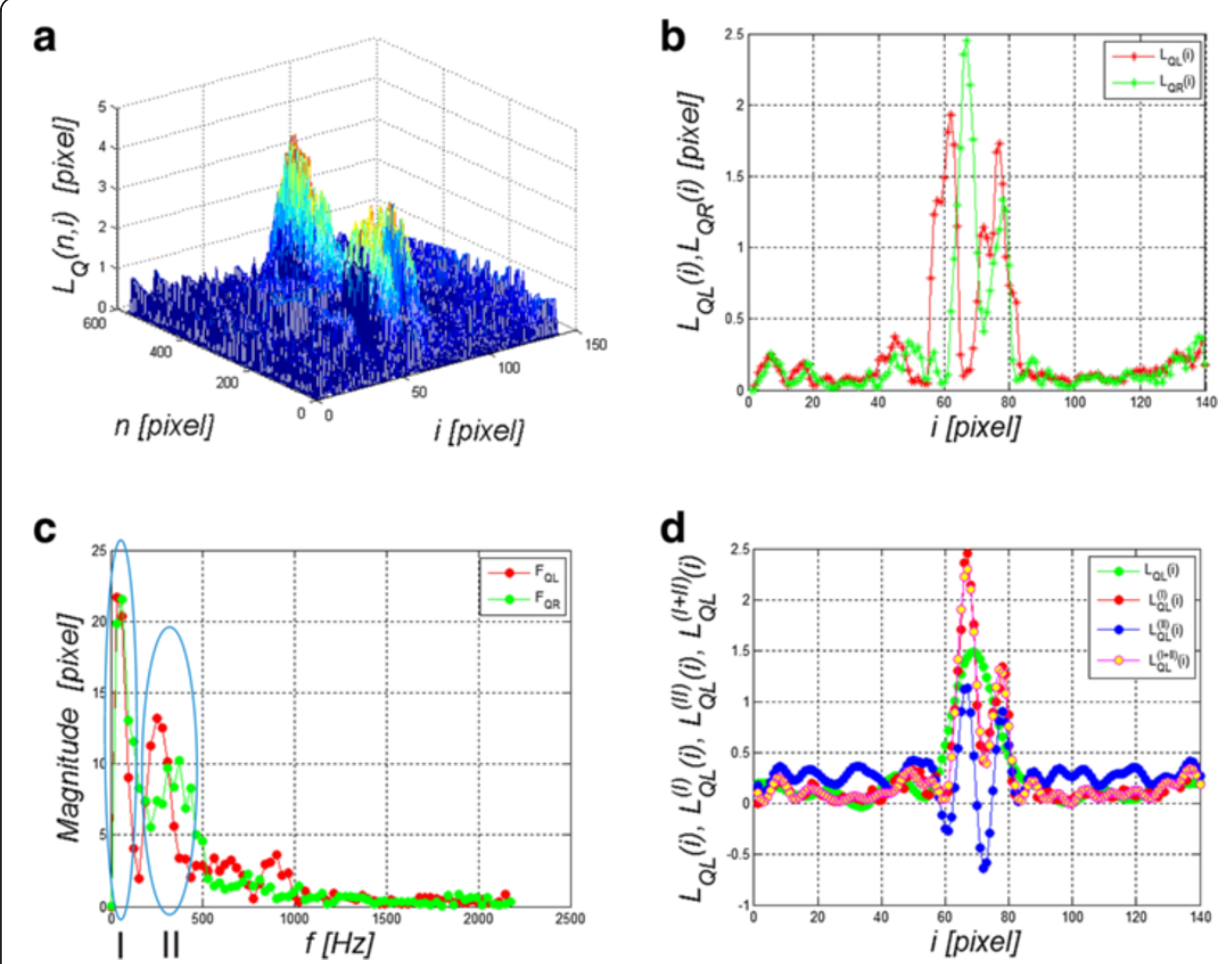

Figure 8 Sample result - the image $L_{Q}(n, i)$ and its frequency analysis. Figure a) shows $L_{Q}(n, i)$, b) shows graphs $L_{Q L}(i)$ and $L_{Q R}(i)$, c) shows frequency analysis $F_{Q R}$ and $F_{Q L}$ of the graphs $L_{Q L}(i)$ and $L_{Q R}(i)$, d) shows individual components $L_{Q L}{ }^{(I)}(i)$ and $L_{Q L}{ }^{(I)}(i)$ and their sum $L_{Q L}{ }^{(I+I)}(i)$ of the waveform $L_{Q L}(i)$. 
rapidly changing (after the above $200 \mathrm{~Hz}$ ) positions of the cornea on the left side relative to the axis of symmetry $\left(L_{Q L}(i)\right)$. The analysis of the graphs $F_{Q R}$ and $F_{Q L}$ (Figure 8c) provides very valuable information regarding the frequency range of the cornea changes under the influence of an air puff. It should be noted that the frequency range for the analysed cases of 96 eyes is 150 to $500 \mathrm{~Hz}$.

A block diagram of the proposed algorithm is shown in Figure 9.

\section{Results}

On the basis of the proposed algorithm, a pattern $L_{V}(n, i)$ of the response of the cornea and the entire eyeball to an air puff was created:

$$
L_{V}(n, i)=L_{T O}(N / 2, i)+\max _{S E 2}\left(\min _{S E 2}\left(L_{T R}(n, i)\right)\right)
$$

The obtained images of 96 eyes were compared to the pattern created for each eye separately:

$$
L_{\Delta}(n, i)=L_{T}(n, i)-L_{V}(n, i)
$$

A sample result, the error $L_{\Delta}(n, i)$, is shown in Figure 10. $L_{\Delta}(n, i)$ is the basis for further analysis. Based on $L_{\Delta}(n, i)$, the following new features are calculated:

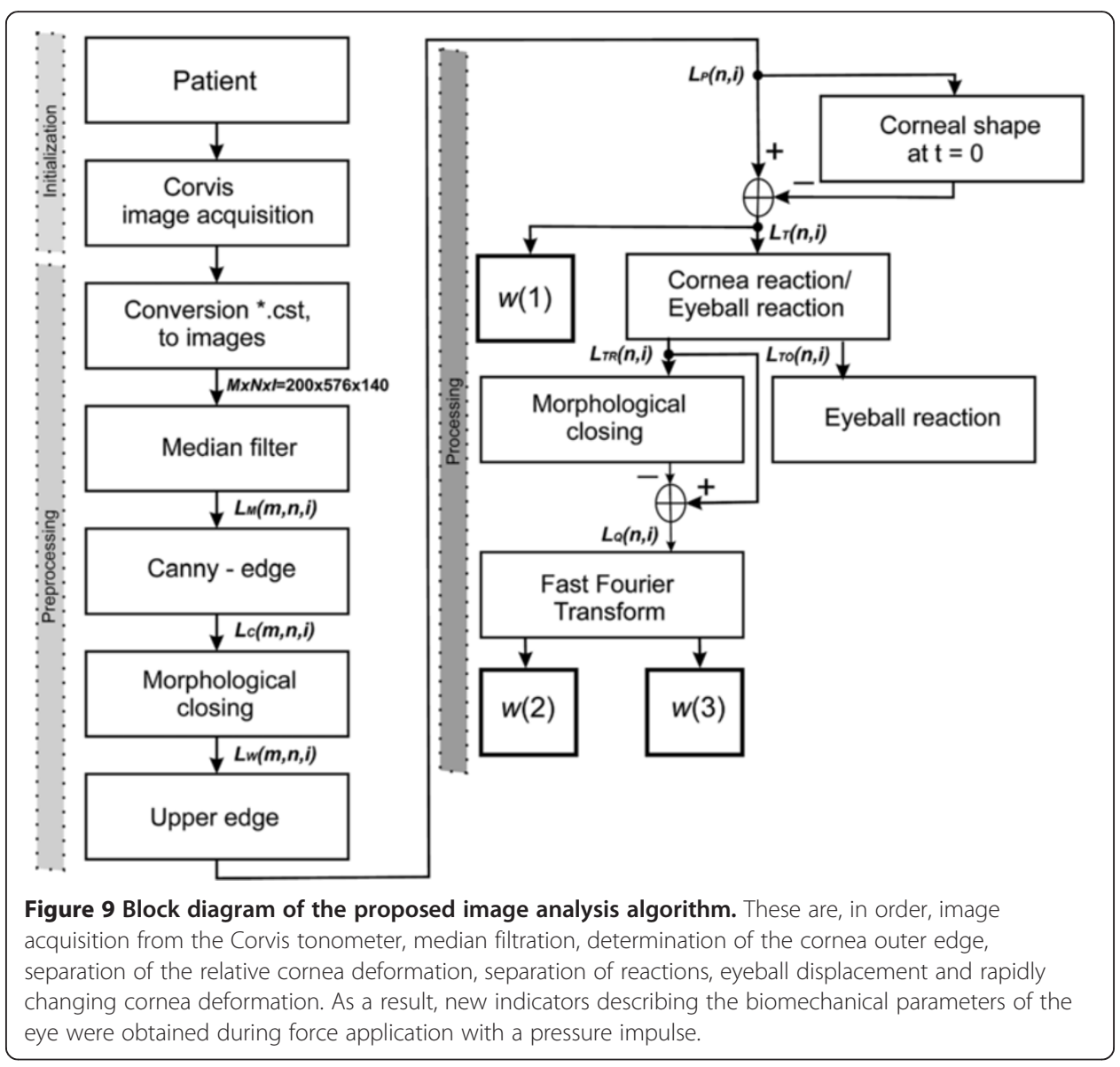




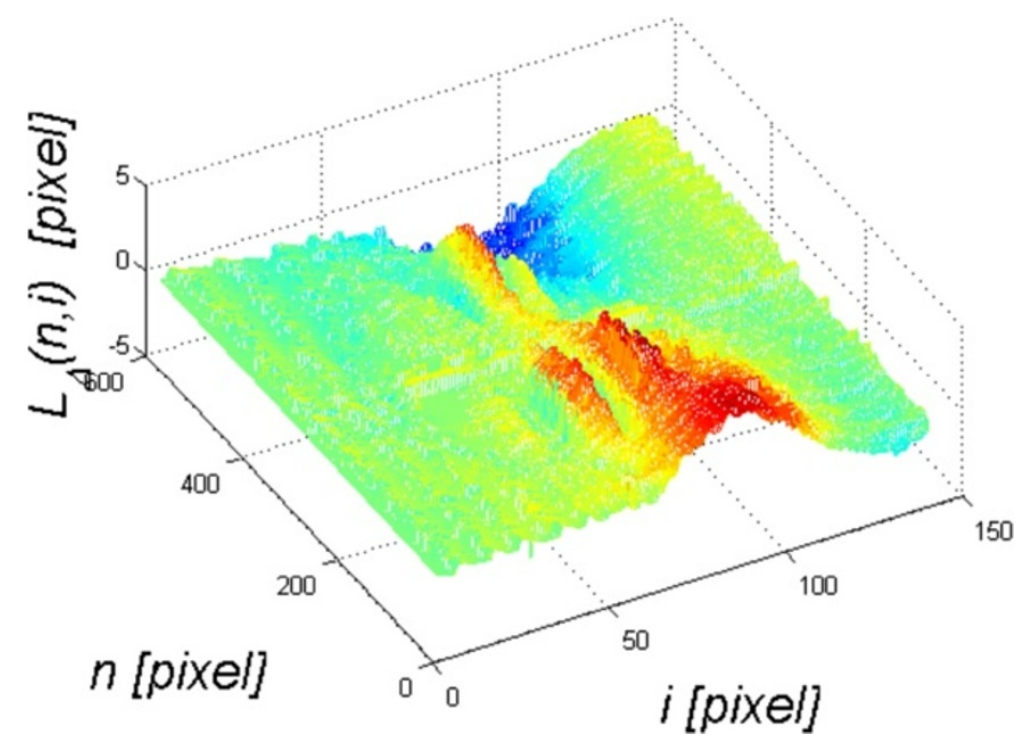

Figure 10 Sample result of the comparison of the registered waveform $L_{T}(n, i)$ with the pattern $L_{V}(n$, i). The resulting error value $L_{\Delta}(n, i)$ is shown above. The rapidly changing cornea deformation during force application and the change in the eyeball position in the eye socket in response to an air-puff stimulus are clearly visible.

$w(1)$ - the maximum amplitude of the eyeball deflection during force application:

$$
w(1)=\max _{i \in(1, I), n \in\{1, N\}}\left(L_{\Delta}(n, i)\right)
$$

$w(2)$ - the maximum amplitude in the spectra $F_{Q R}$ and $F_{Q L}$ for the frequency range from 150 to $500 \mathrm{~Hz}$. With a fixed number of frames per second during registration with the Corvis tonometer (one frame every $23 \mu \mathrm{s}$ ), it is:

$$
w(2)=\max _{i \in(7,15)}\left(F_{Q R}(i), F_{Q L}(i)\right)
$$

$w(3)$ - the duration of the rapidly changing (from 150 to $500 \mathrm{~Hz}$ ) cornea deformations calculated as a time range with a threshold of $80 \%$ of the maximum amplitude Figure 10.

The choice of features and their number is closely related to the previously described automatically calculated parameters such as the correction of the cornea deformation or the entire eyeball reaction to a stimulus. The obtained results, the values of $w(1)$, $w(2)$ and $w(3)$, measured with the Corvis tonometer for the first 10 eyes are shown in Table 1. The correlation results of the features $w(1)$ and $w(2)$ with $w(3)$ are shown in Figure 11. The results presented in Figure 11 were divided into 4 different classes. The adopted number of classes is the result of the analysis of the distribution of individual clusters forming sets that do not have common features. On this basis, a decision tree was created which is shown in Figure 12. In all cases, a non-parametrical algorithm CART (Classification and Regression Trees) creating binary trees is used as the method for their induction. An increase in the nodes purity has been used as the criterion for assessing the quality of CART divisions. The Gini index has been used as the measure of nodes impurity. Because of a small number of cases, the tree creation was not limited by a minimum number of vectors in a node. In its current form (Figure 12), there 
Table 1 Summary of sample features $w(1), w(2)$ and $w(3)$ for 10 eyes (only the right ones) and their division into classes according to the proposed classifier

\begin{tabular}{lllll}
\hline $\mathbf{N o}$ & $\boldsymbol{w ( 1 )}$ & $\boldsymbol{w}(\mathbf{2})$ & $\boldsymbol{w}(\mathbf{3})$ & Class \\
\hline $\mathbf{1}$ & 37.2 & 2.5 & 17.0 & 1 \\
$\mathbf{2}$ & 15.0 & 3.2 & 16.8 & 2 \\
$\mathbf{3}$ & 13.1 & 4.3 & 22.0 & 2 \\
$\mathbf{4}$ & 25.6 & 5.0 & 19.5 & 2 \\
$\mathbf{5}$ & 28.6 & 28.6 & 3 \\
$\mathbf{6}$ & 20.2 & 16.3 & 30.6 & 3 \\
$\mathbf{7}$ & 13.9 & 3.2 & 24.2 & 1 \\
$\mathbf{8}$ & 20.3 & 25.1 & 4 \\
$\mathbf{9}$ & 38.2 & 25.5 & 25.6 & 3 \\
$\mathbf{1 0}$ & 48.4 & 5.2 & 23.9 & 2 \\
\hline
\end{tabular}

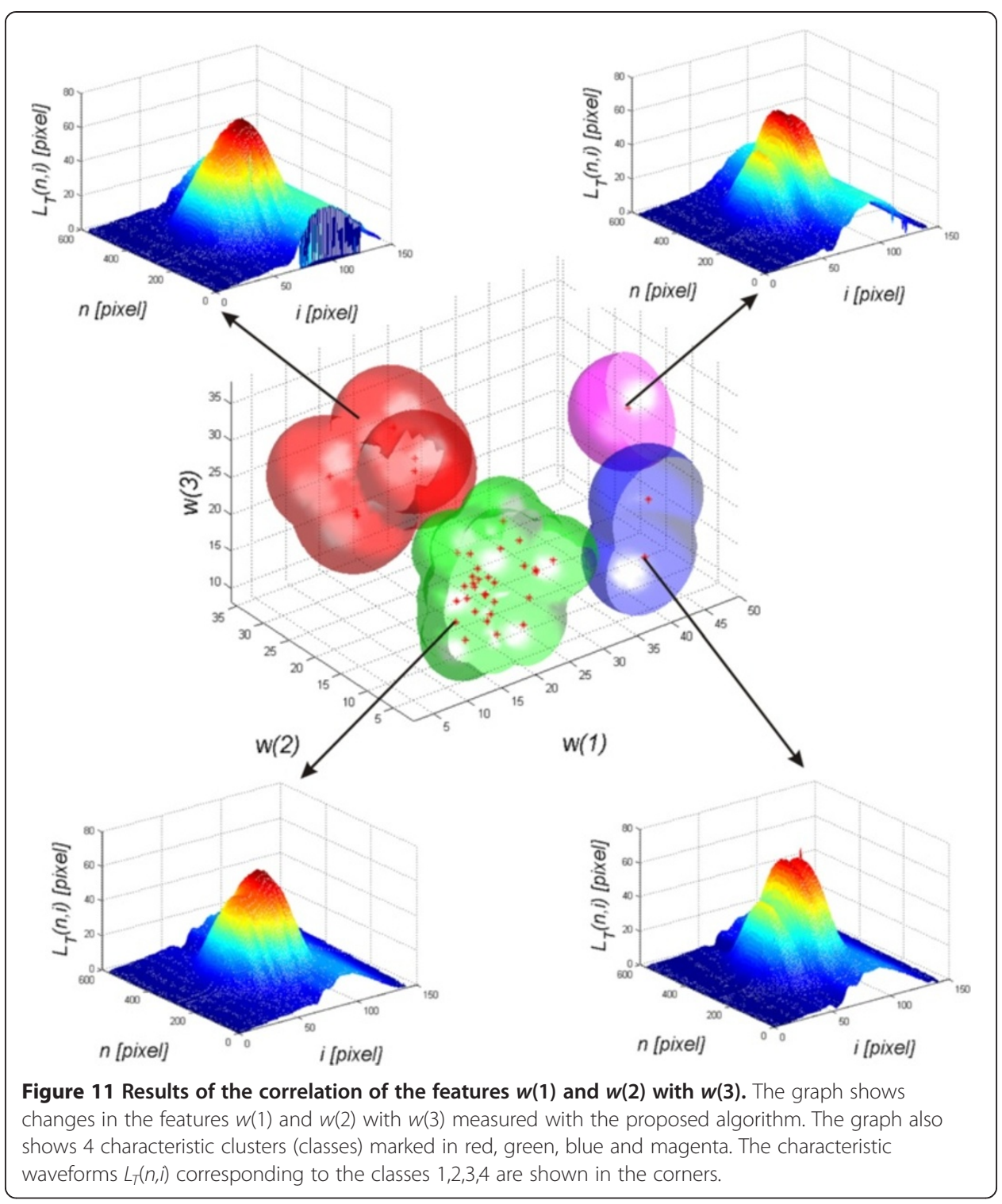




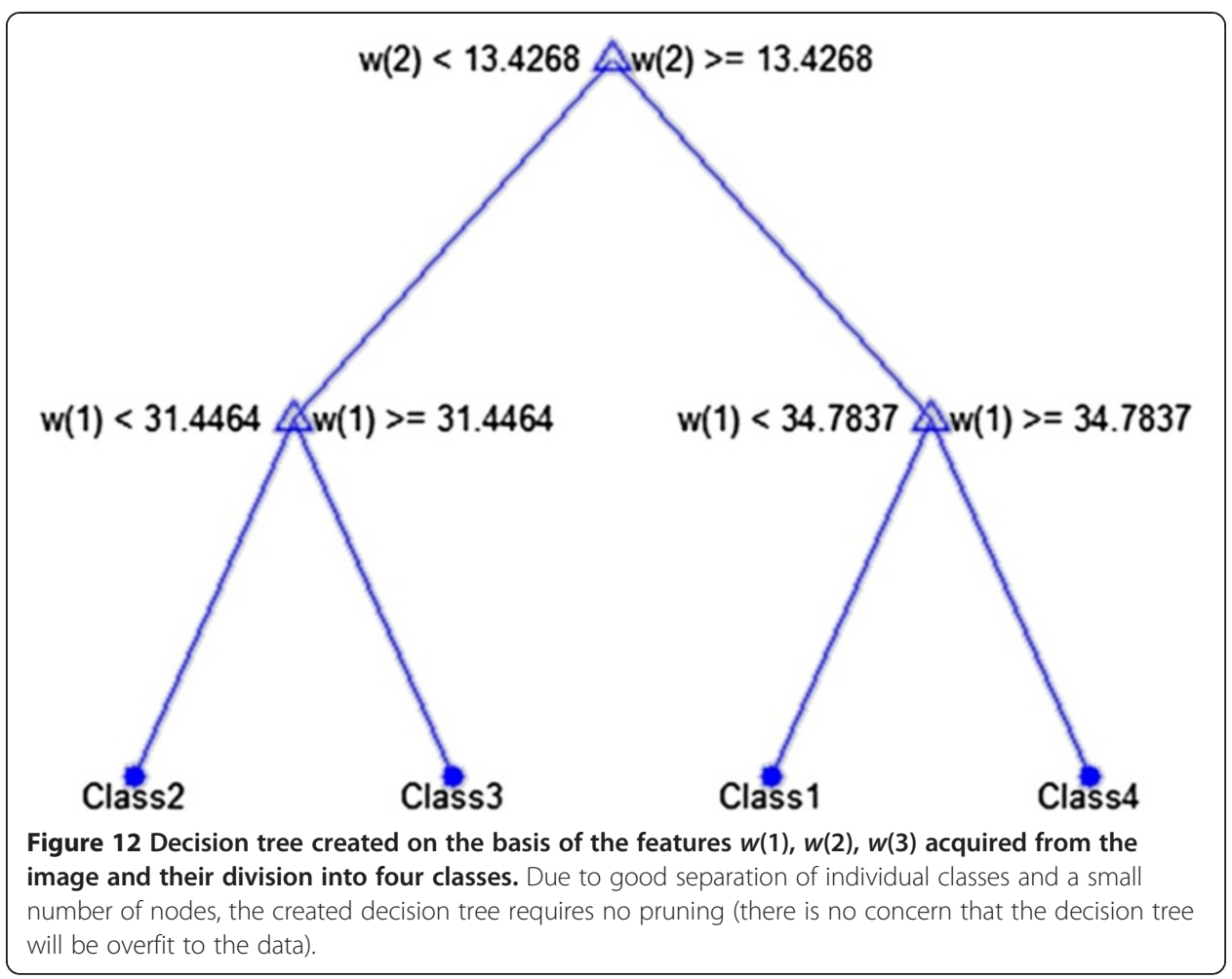

is no need to trim the tree - there is no concern that the decision tree will be overfit to the data. For the analysed number of cases, all classification results are correct. The number of the eyes classified as Class 1 is 6 (Figure 11 - red), Class 3-2 (Figure 11 blue), Class $4-2$ (Figure 11 magenta), and the rest belongs to Class 2 (Figure 11 green). An increase in the number of the analysed eyes (patients) may, in the future, give more reliable results and provide additional evidence as to the number of classes. The individual classes, depending on the range of feature values $(w(1), w(2)$ and $w(3))$ which is characteristic for them, define various types of reactions of the entire eyeball to a stimulus and different amplitudes of rapidly changing deformations of the cornea itself. A sample graph of the dependence of the maximum displacement of the entire eyeball $w(1)$ on the cornea deformation $\max \left(L_{T R}\right)$ for each class is shown in Figure 13. Both the features as well as the division into classes can be further used not only as an additional diagnostic tool but they can also enrich the existing software of the Corvis tonometer.

\section{Critical summary}

The paper presents the possibility of using the profiled algorithm of image analysis, proposed by the authors, to measure additional parameters of the corneal deformation. This algorithm enables separation of the eyeball reaction from the corneal deflection during force application. In addition, measurements of rapidly changing cornea deformations (from 150 to $500 \mathrm{~Hz}$ ) were proposed. These measurements allowed to propose a pattern individually for each eye. The pattern, in turn, helped to determine deviations individually for each eye. On the basis of these deviations, 


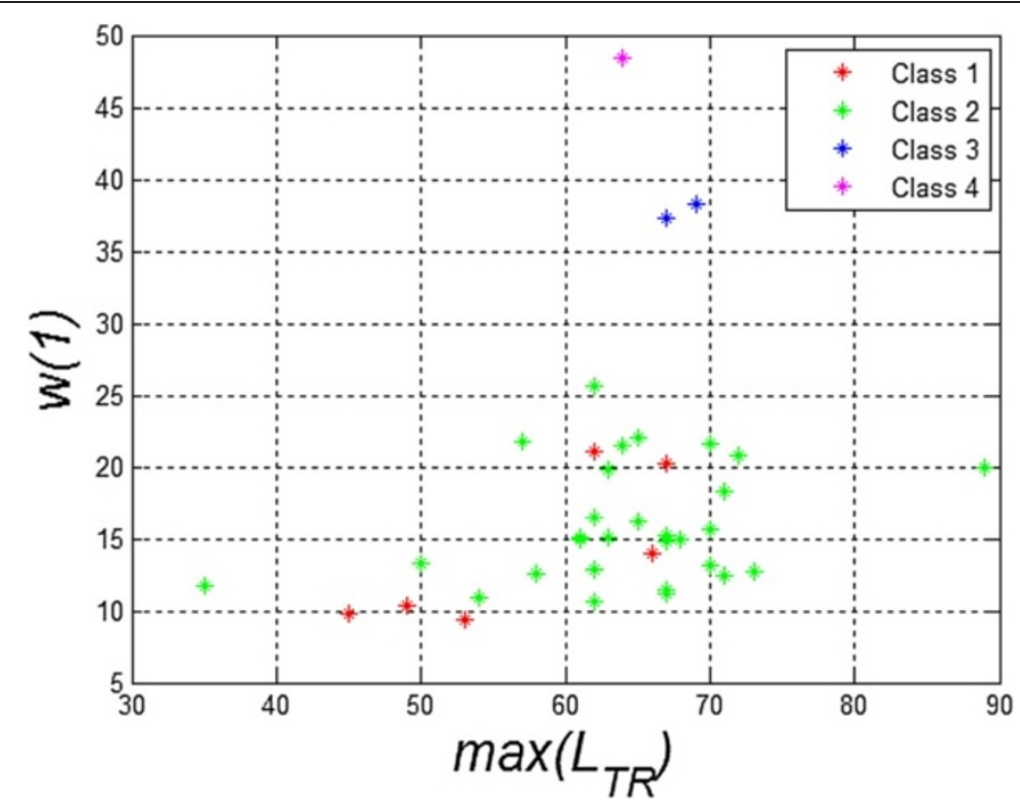

Figure 13 Graph showing the dependence of the maximum eyeball displacement $w(1)$ on the cornea deformation $\max \left(L_{T R}\right)$ for each class. The graph results from the division of deformations automatically measured with the proposed algorithm into 4 classes. The results obtained provide the basis for further analysis and comparison of biomechanical parameters of the eye in future studies.

the features $w(1), w(2)$ and $w(3)$ as well as the classifier (decision tree) allowing a division into four classes were calculated.

The paper only presents a tool for automatic measurement of additional new parameters when using the Corvis tonometer. A detailed clinical examination based on this method will be presented in subsequent papers. In addition, the accuracy and repeatability of measurements for one patient and a phantom, the correlation of features (primarily $w(1))$ for a single patient and the right and left eyes and the effect of the patient position during the test on the result, both IOP as well as the other features, will be verified. The issue of analysis of symmetry, or its lack, in the dynamic response of the entire eyeball to an air-puff stimulus, is quite interesting and remains open. The analysis of corneal deflection parallel in other cross-sections is an open topic here. From a practical point of view, higher harmonics (above $2 \mathrm{kHz}$ ), that for the current sampling frequency (every $23 \mu \mathrm{s}$ ) are difficult to analyse, are also interesting. Moreover, the presented methods of image analysis and processing may be carried out in another way, in another dedicated algorithm, for example, with the use of random methods of analysis of layers in tomographic eye images [26,27], the methods of mathematical morphology [28] or others [29]. The final choice of the analysis method is highly dependent on the results obtained in practice, which is an important element given the large interindividual variability. Indeed, biomechanical parameters of the cornea, measured in dynamic states, still represent a new area of interdisciplinary research.

\section{Abbreviations}

ROI: Region Of Interest; IOP: Intraocular Pressure; FFT: Fast Fourier Transform; LASIK: Laser-Assisted in situ Keratomileusis. 
Authors' contributions

RK suggested the algorithm for image analysis and processing, implemented it and analysed the images, wrote the full text of the manuscript. AL, AN, EW, ZW performed the acquisition the images from Corvis and HK consulted the obtained results. All authors have read and approved the final manuscript.

\author{
Acknowledgements \\ No outside funding was received for this study.
}

\title{
Author details
}

'Department of Biomedical Computer Systems, University of Silesia, Faculty of Computer Science and Materials Science, Institute of Computer Science, ul. Będzińska 39, Sosnowiec 41-200, Poland. ${ }^{2}$ Ophthalmology Clinic, Medical University of Silesia, District Railway Hospital in Katowice, Katowice, Poland. ${ }^{3}$ Institute of Physics, Wroclaw University of Technology, Wybrzeze Wyspianskiego 27, Wroclaw 50-370, Poland.

Received: 19 March 2014 Accepted: 29 April 2014

Published: 3 May 2014

\section{References}

1. Luce DA: Determining in vivo biomechanical properties of the cornea with an ocular response analyzer. $J$ Cataract Refract Surg 2005, 31(1):156-162.

2. Kobayashi AS, Staberg LG, Schlegel WA: Viscoelastic properties of human cornea. Exp Mech 1973, 12:497-503.

3. Malik NS, Moss SJ, Ahmed N, Furth AJ, Wall RS, Meek KM: Ageing of the human corneal stroma: structural and biochemical changes. Biochim Biophys Acta 1992, 1138(3):222-228.

4. Sherrard ES, Novakovic P, Speedwell L: Age-related changes of the corneal endothelium and stroma as seen in vivo by specular microscopy. Eye (Lond) 1987, 1(Pt2):197-203.

5. Kotecha A, Elsheikh A, Roberts CR, Zhu HG, Garway-Heath DF: Corneal thicknessand age-related biomechanical properties of the cornea measured with the ocular response analyzer. Invest Ophthalmol Vis Sci 2006, 47(12):5337-5347.

6. Valbon BF, Ambrósio-Jr R, Fontes BM, Alves MR: Effects of age on corneal deformation by non-contact tonometry integrated with an ultra-high-speed (UHS) Scheimpflug camera. Ara Bras Oftalmol 2013, 76(4):229-232.

7. Brown KE, Congdon NG: Corneal structure and biomechanics: impact on the diagnosis and management of glaucoma. Curr Opin Ophthalmol 2006, 17(4):338-343.

8. Dupps WJ, Wilson SE: Biomechanics and wound healing in the cornea. Exp Eye Res 2006, 83(4):709-720.

9. Elsheikh A, Alhasso D, Rama P: Assessment of the epithelium's contribution to corneal biomechanics. Exp Eye Res 2008, 86(2):445-451.

10. Meek KM, Leonard DW: Ultrastructure of the corneal stroma: a comparative study. Biophys J 1993, 64(1):273-280.

11. Fontes BM, Ambrosio R Jr, Alonso RS, Jardim D, Velarde GC, Nose W: Corneal biomechanical metrics in eyes with refraction of -19.00 to +9.00 D in healthy brazilian patients. J Refract Surg 2008, 24(9):941-945.

12. Kirwan C, O'Keefe M, Lanigan B: Corneal hysteresis and intraocular pressure measurement in children using the Reichert ocular response analyzer. Am J Ophthalmol 2006, 142(6):990-992.

13. Shah S, Laiquzzaman M, Bhojwani R, Mantry S, Cunliffe I: Assessment of the biomechanical properties of the cornea with the ocular response analyzer in normal and keratoconic eyes. Invest Ophthalmol Vis Sci 2007, 48(7):3026-3031.

14. Touboul D, Roberts C, Kerautret J, Garra C, Maurice-Tison S, Saubusse E, Colin J: Correlation between corneal hysteresis intraocular pressure, and corneal central pachymetry. J Cataract Refract Surg 2008, 34(4):616-622.

15. Congdon NG, Broman AT, Bandeen-Roche K, Grover D, Quigley HA: Central corneal thickness and corneal hysteresis associated with glaucoma damage. Am J Ophthalmol 2006, 141(5):868-875.

16. Gatinel D, Chaabouni S, Adam PA, Munck J, Puech M, Hoang-Xuan T: Corneal hysteresis, resistance factor, topography, and pachymetry fter corneal lamellar flap. J Refract Surg 2007, 23(1):76-84.

17. Shah S, Laiquzzaman M, Cunliffe I, Mantry S: The use of the Reichert ocular response analyser to establish the relationship between ocular hysteresis, corneal resistance factor and central corneal thickness in normal eyes. Cont Lens Anterior Eye 2006, 29(5):257-262.

18. Ortiz D, Pinero D, Shabayek MH, Arnalich-Montiel F, Alió JL: Corneal biomechanical properties in normal, post-laser in situ keratomileusis, and keratoconic eyes. J Cataract Refract Surg 2007, 33(8):1371-1375.

19. Pepose JS, Feigenbaum SK, Qazi MA, Sanderson JP, Roberts CJ: Changes in corneal biomechanics and intraocular pressure following LASIK using static, dynamic, and noncontact tonometry. Am J Ophthalmol 2007 143(1):39-47.

20. Elsheikh A, Anderson K: Comparative study of corneal strip extensometry and inflation tests. J R Soc Interface 2005, 2(3):177-185.

21. Reznicek L, Muth D, Kampik A, Neubauer AS, Hirneiss C: Evaluation of a novel Scheimpflug-based non-contact tonometer in healthy subjects and patents with ocular hypertension and glaucoma. Br J Ophthalmol 2013, 97(11):1410-1414.

22. Hon Y, Lam AKC: Corneal deformation measurement using scheimpflug noncontact tonometer. Optometry \& Vistion Science 2013, 90(1):e1-e8.

23. Leung CKS, Ye C, Weinreb RN: An ultra high-speed Scheimpflug camera for evaluation of corneal deformation response and its impact on IOP measurement. Investigative Ophtalmology \& Visual Science 2013, 54(4):2885-2892.

24. Kling S, Marcos S: Contributing factors to corneal deformation in air puff measurements. Investigative Ophtalmology \& Visual Science 2013, 54(7):5078-5085.

25. Otsu N: A threshold selection method from gray-level histograms. IEEE Trans Sys, Man, Cyber 1979, 9(1):62-66

26. Koprowski R, Wrobel Z: Identification of layers in a tomographic image of an eye based on the canny edge detection. Inf Technol Biomed Adv Intell Soft Comput 2008, 47:232-239. 
27. Koprowski R, Wróbel Z: Layers recognition in tomographic eye image based on random contour analysis. Computer recognition systems 3. Adv Intell Soft Comput 2009, 57:471-478.

28. Korzynska A, Iwanowski M: Multistage morphological segmentation of bright-field and fluorescent microscopy images. Opt-Electron Rev 2012, 20(2):87-99.

29. Wilczyński S, Ptaszkiewicz M, Pierzchała E, Pilawa B, Swakoń J, Olko P: Electron paramagnetic resonance studies of gamma irradiated azithromycin. Current Topics in Biophysics 2008, 31:1-4.

doi:10.1186/1475-925X-13-55

Cite this article as: Koprowski et al: Selected parameters of the corneal deformation in the Corvis tonometer. BioMedical Engineering OnLine 2014 13:55.

\section{Submit your next manuscript to BioMed Central} and take full advantage of:

- Convenient online submission

- Thorough peer review

- No space constraints or color figure charges

- Immediate publication on acceptance

- Inclusion in PubMed, CAS, Scopus and Google Scholar

- Research which is freely available for redistribution

Submit your manuscript at www.biomedcentral.com/submit

() Biomed Central 